\title{
THE CORONA THEOREM FOR DOMAINS WHOSE BOUNDARY LIES IN A SMOOTH CURVE
}

\author{
CHARLES N. MOORE
}

\begin{abstract}
We prove the corona theorem for domains whose boundary lies in a $C^{1+\alpha}$ curve.
\end{abstract}

Let $\Gamma$ be a $C^{1+\alpha}$ Jordan curve in the plane $\mathbf{C}$ and let $E$ be a compact subset of $\Gamma$. Set $\Omega=\mathbf{C}-E$, and let $H^{\infty}(\Omega)$ denote the space of bounded analytic functions on $\Omega$. We show:

THEOREM. Let $f_{1}, \ldots, f_{n} \in H^{\infty}(\Omega)$ and $\mu>0$ have the property that $\mu \leq$ $\max \left\{\left|f_{j}(z)\right|: 1 \leq j \leq n\right\} \leq 1$ for every $z \in \Omega$. Then there exists $g_{1}, \ldots, g_{n} \in$ $H^{\infty}(\Omega)$ such that $f_{1} g_{1}+\cdots+f_{n} g_{n} \equiv 1$ on $\Omega$.

When $\Gamma$ is the real line, such a domain $\Omega$ is called a Denjoy domain. In this case the theorem has been proven by Garnett and Jones $[3]$; the proof of the above theorem will be based on their result.

To prove the theorem, it suffices to do it locally, that is, we show that for $\varsigma \in \Gamma$ there exists a neighborhood of $\zeta$ on which the theorem is true and such that the size of this neighborhood is determined only by $\mu, n$, and parameters concerning $\Gamma$. The fact that we can localize the problem is due to Gamelin [1], see also Garnett [2, p. 368].

We may assume $\Gamma$ has length 1 . Let $\varsigma(s):[0,1] \rightarrow \mathbf{C}$ be a parametrization of $\Gamma$ by arc length. Since $\Gamma$ is $C^{1+\alpha}$, there exists a constant $c_{1}>0$ such that $\left|\zeta^{\prime}(s)-\zeta^{\prime}(t)\right| \leq c_{1}|s-t|^{\alpha}$ for all $s, t \in[0,1]$. At this point we note that with just minor modification the proof works if we only assume $\zeta^{\prime}(s)$ satisfies a Dini condition, $\int_{0}^{1} \omega(\delta) / \delta d \delta<\infty$, where $\omega(\delta)$ is the modulus of continuity of $\zeta^{\prime}(s)$. However, the idea of the proof is amply illustrated by the $C^{1+\alpha}$ case and this is the only case we consider.

The proof will be divided into three lemmas, the first of which is the crux of the proof. This lemma says that locally on a $C^{1+\alpha}$ curve, Cauchy integrals behave essentially like Cauchy integrals along straight lines. The proof of this lemma is similar to the proof of the fact that the Cauchy integral is a bounded operator on $L^{2}$ on a $C^{1+\alpha}$ curve. Lemma 2 will then show that locally, $H^{\infty}(\Omega)$ functions are close to $H^{\infty}$ functions on Denjoy domains. Then Lemma 3 shows how to obtain a local solution given that the problem can be solved on Denjoy domains.

The author would like to thank Professors S.-Y. A. Chang and J. B. Garnett for many helpful conversations during the preparation of this paper.

The letter $c$ will denote a constant, not necesarily the same at each occurrence. All such constants will depend only on the curve $\Gamma$ and the numbers $n$ and $\mu$.

Received by the editors April 17, 1986.

1980 Mathematics Subject Classification (1985 Revision). Primary 30D50.

The author was supported by NSF Grant DMS-85-03780 
For $z=x+i y \in \mathbf{C}$ with $x \in[0,1]$ we define $T z=\varsigma(x)+i \zeta^{\prime}(x) y$.

Lemma 1. Let $u(s) \in L^{\infty}([0,1])$. Then there exists an $\varepsilon>0$ such that if $I$ is any interval in $[0,1]$ with $|I|<\varepsilon$, and if $z=x+i y$ with $x \in I$, then

$$
\left|\frac{1}{\zeta^{\prime}(x)} \int_{I} \frac{u(s)}{s-z} d s-\int_{I} \frac{u(s)}{\zeta(s)-T z} d s\right| \leq c\|u\|_{\infty}|I|^{\alpha} .
$$

ProOF. First note that

$$
\begin{aligned}
\left|\zeta(s)-\zeta(x)-\zeta^{\prime}(x)(s-x)\right| & =\left|\int_{x}^{s} \varsigma^{\prime}(t) d t-\zeta^{\prime}(x) \int_{x}^{s} 1 d t\right| \\
& \leq c \int_{0}^{|s-x|} t^{\alpha} d t=\frac{c}{\alpha+1}|s-x|^{\alpha+1} .
\end{aligned}
$$

Then, also,

$$
\left|\frac{\zeta(s)-\zeta(x)}{s-x}-\varsigma^{\prime}(x)\right| \leq \frac{c}{\alpha+1}|s-x|^{\alpha} .
$$

Choose $\varepsilon$ so that $(c /(\alpha+1))|s-x|^{\alpha} \leq 1 / 2$ for every $s, x \in I$. Then,

$$
\begin{aligned}
& \left|\frac{1}{\zeta^{\prime}(x)} \frac{1}{s-z}-\frac{1}{\zeta(s)-T z}\right| \\
& \quad=\left|\frac{\zeta(s)-\zeta(x)-\zeta^{\prime}(x)(s-x)}{\zeta^{\prime}(x)(s-x-i y)\left(\zeta(s)-\zeta(x)-i y \zeta^{\prime}(x)\right)}\right| \\
& \quad \leq \frac{c|s-x|^{\alpha+1}}{|s-x-i y|\left|\zeta(s)-\zeta(x)-i y \zeta^{\prime}(x)\right|} \\
& \quad \leq \frac{c|s-x|^{\alpha-1}}{|1-i y /(s-x)|(|1-i y /(s-x)|-1 / 2)} \leq c|s-x|^{\alpha-1} .
\end{aligned}
$$

Then the left-hand side of (1) can be estimated by

$$
\int_{I} c|s-x|^{\alpha-1}|| u\left\|_{\infty} d s \leq c\right\| u \|_{\infty}|I|^{\alpha}
$$

which proves Lemma 1.

Let $s_{0} \in[0,1]$ so that $\zeta\left(s_{0}\right) \in \Gamma$. We want to define a region $D$ near $s_{0}$, and a region $\tilde{D}$ near $\zeta\left(s_{0}\right)$ and compare $H^{\infty}$ functions on the two regions.

There exists a number $\delta_{0}>0$ which depends only on $\Gamma$ such that for all $\delta$ with $|\delta|<\delta_{0}$ we have $\zeta(x)+i \delta \zeta^{\prime}(x) \in \Gamma$ only when $\delta=0$. This follows from the compactness of $[0,1]$ and the fact that $\Gamma$ is a Jordan curve.

Let $I=[a, b]$ be an interval centered at $s_{0}$ and let

$$
\beta=\sup \left\{\left|\varsigma^{\prime}(s)-\varsigma^{\prime}(t)\right|: s, t \in I\right\} .
$$

We can assume that $I$ is chosen small enough so that $\beta<1 / 10$; this insures us that up to a rotation, $\varsigma(I)$ is the graph of a function. We also suppose that $|I|<\varepsilon$, where $\varepsilon$ is from Lemma 1 .

Now define

$$
D=\{x+i y: a<x<b,|y|<\delta\}-\varsigma^{-1}(E)
$$

and

$$
\tilde{D}=\left\{\varsigma(s)+i n \varsigma^{\prime}\left(s_{0}\right): s \in I,|n|<\delta\right\}-E,
$$


where $\delta<\delta_{0}$ is fixed but chosen later. Let $\partial D=\gamma$ and $\partial \tilde{D}=\tilde{\gamma}$; it will be necessary to label different parts of $\gamma$ and $\tilde{\gamma}$. Set

$$
\begin{aligned}
& \gamma_{1}=\{a+i n:|n|<\delta\} \cup\{b+i n:|n| \leq \delta\}, \\
& \gamma_{2}=\{s+i \delta: s \in I\}, \quad \gamma_{3}=\{s-i \delta: s \in I\} .
\end{aligned}
$$

Analogously,

$$
\begin{aligned}
& \tilde{\gamma}_{1}=\left\{\varsigma(a)+i n \zeta^{\prime}\left(s_{0}\right):|n| \leq \delta\right\} \cup\left\{\zeta(b)+i n \varsigma^{\prime}\left(s_{0}\right):|n| \leq \delta\right\}, \\
& \tilde{\gamma}_{2}=\left\{\varsigma(s)+i \delta \zeta^{\prime}\left(s_{0}\right): s \in I\right\}, \quad \tilde{\gamma}_{3}=\left\{\zeta(s)-i \delta \zeta^{\prime}\left(s_{0}\right): s \in I\right\}, \\
& \text { so } \gamma=\left(I \cap \zeta^{-1}(E)\right) \cup \gamma_{1} \cup \gamma_{2} \cup \gamma_{3} \text { and } \gamma=(\zeta(I) \cap E) \cup \tilde{\gamma}_{1} \cup \tilde{\gamma}_{2} \cup \tilde{\gamma}_{3} \text {. }
\end{aligned}
$$

Define a map $\omega(\xi): \gamma \rightarrow \tilde{\gamma}$ by

$$
\omega(\xi)= \begin{cases}\varsigma(s) & \text { if } \xi=s \in \varsigma^{-1}(E), \\ \varsigma(a)+i n \varsigma^{\prime}\left(s_{0}\right) & \text { if } \xi=a+i n \\ \varsigma(b)+i n \varsigma^{\prime}\left(s_{0}\right) & \text { if } \xi=b+i n \\ \varsigma(s)+i \delta \zeta^{\prime}\left(s_{0}\right) & \text { if } \xi=s+i \delta \\ \varsigma(s)-i \delta \varsigma^{\prime}\left(s_{0}\right) & \text { if } \xi=s-i \delta\end{cases}
$$

When integrating over $\gamma$ and $\tilde{\gamma}$ we will also consider them oriented as follows: $\gamma_{1} \cup \gamma_{2} \cup \gamma_{3}$ is a square, orient it in a counterclockwise direction, and give $I \cap \zeta^{-1}(E)$ its orientation from the real line. Then give $\tilde{\gamma}$ the induced orientation from $\gamma$ via $\omega(\xi)$. Also we define

$$
D_{1 / 2}=\left\{x+i y:\left|x-s_{0}\right|<\frac{b-a}{4},|y|<\frac{\delta}{2}\right\}-\varsigma^{-1}(E) .
$$

Then we have

LEMMA 2. If $h(\xi) \in L^{\infty}(\gamma)$ and $z=x+i y \in D_{1 / 2}$, then

$$
\left|\frac{1}{\zeta^{\prime}(x)} \int_{\gamma} \frac{h(\xi)}{\xi-z} d \xi-\int_{\gamma} \frac{h(\xi)}{\omega(\xi)-T z} d \xi\right| \leq c|| h \|_{\infty}\left(|I|^{\alpha}+\frac{\delta}{|I|}+\frac{|I|}{\delta} \beta\right) .
$$

ProOF. We estimate this difference by estimating on each piece of $\gamma$. On $I \cap \varsigma^{-1}(E), \xi=s, \omega(\xi)=\varsigma(s)$ and the contribution to $(2)$ is

$$
\left|\frac{1}{\zeta^{\prime}(x)} \int_{I \cap \zeta^{-1}(E)} \frac{h(s)}{s-z} d s-\int_{I \cap \varsigma^{-1}(E)} \frac{h(s)}{\zeta(s)-T z} d s\right| \leq c|| h \|_{\infty}|I|^{\alpha}
$$

by Lemma 1 .

For $\xi \in \gamma_{1}$ we have $\xi=a+i s$ and $\xi=b+i s$, where $|s| \leq \delta$, and so $\omega(\xi)=$ $\varsigma(a)+i s \zeta^{\prime}\left(s_{0}\right)$ and $\omega(\xi)=\varsigma(b)+i s \zeta^{\prime}\left(s_{0}\right),|s| \leq \delta$. Thus,

$$
\begin{gathered}
\left|\frac{1}{\zeta^{\prime}(x)} \int_{\gamma_{1}} \frac{h(\xi)}{\xi-z} d \xi\right| \leq \frac{16 \delta\|h\|_{\infty}}{|I|}=\frac{c \delta\|h\|_{\infty}}{|I|}, \\
\left|\int_{\gamma_{1}} \frac{h(\xi)}{\omega(\xi)-T z} d \xi\right| \leq \frac{c \delta\|h\|_{\infty}}{|I|}
\end{gathered}
$$

where, in (5), $c$ depends on $\beta$, but is bounded for all $\beta<1 / 10$.

It remains to estimate the contribution to the left side of (2) by the integrals over $\gamma_{2}$ and $\gamma_{3}$. We just estimate the integral over $\gamma_{2}$; the estimate of the integral 
over $\gamma_{3}$ is similar. On $\gamma_{2}, \xi(s)=s+i \delta, s \in I$, and $\omega(\xi)=\varsigma(s)+i \delta \varsigma^{\prime}\left(s_{0}\right), s \in I$, that is, $w(\xi(s))$ is just a translation of $\varsigma(s)$ by $i \delta^{\prime}\left(s_{0}\right)$. Note that we can neglect the orientation we have given $\gamma_{2}$ since it is only the modulus of an integral that we wish to estimate. For $z=x+i y$ with $x \in I$, set

$$
S z=\omega(\xi(x))+i\left(\frac{d(\omega \circ \xi)}{d s}(x)\right)(y-\delta)=\varsigma(x)+i \delta \varsigma^{\prime}\left(s_{0}\right)+i \varsigma^{\prime}(x)(y-\delta) .
$$

Then by Lemma 1 ,

$$
\left|\frac{1}{\zeta^{\prime}(x)} \int_{I} \frac{h(s+i \delta)}{s+i \delta-z} d s-\int_{I} \frac{h(s+i \delta)}{\zeta(s)+i \delta \zeta^{\prime}\left(s_{0}\right)-S z} d s\right| \leq c|| h \|_{\infty}|I|^{\alpha} .
$$

Noting that $|S z-T z| \leq \beta \delta$ we also have

$$
\left|\int_{\gamma_{2}} \frac{h(\xi)}{\omega(\xi)-S z} d \xi-\int_{\gamma_{2}} \frac{h(\xi)}{\omega(\xi)-T z} d \xi\right| \leq \frac{c\|h\|_{\infty}}{\delta} \beta|I| .
$$

Then (6) and (7) yield an estimate for $\gamma_{2}$. This estimate together with a similar estimate for $\gamma_{3}$ and (3), (4), and (5) give (2).

The theorem will then follow from

LEMMA 3. There exists a neighborhood $U$ of $\varsigma\left(s_{0}\right)$ and $g_{1}, \ldots, g_{n} \in H^{\infty}(U)$ such that $f_{1} g_{1}+\cdots+f_{n} g_{n} \equiv 1$ on $U$.

ProOF. For $\xi \in \Gamma$ and $1 \leq j \leq n$ we let

$$
J f_{j}(\xi)=\lim _{\substack{\omega \rightarrow \xi \\ \omega \in \Gamma_{\xi}^{+}}} f_{j}(\omega)-\lim _{\substack{\omega \rightarrow \xi \\ \omega \in \Gamma_{\xi}^{-}}} f_{j}(\omega)
$$

where $\Gamma_{\xi}^{+}, \Gamma_{\xi}^{-}$are cones at $\xi \in \Gamma$ centered along the positive and negative normals at $\xi$, with apertures chosen so that they lie completely on one side of $\Gamma$. Set

$$
F_{j}(\omega)= \begin{cases}f_{j}(\omega) & \text { if } \omega \in \tilde{\gamma}_{1} \cup \tilde{\gamma}_{2} \cup \tilde{\gamma}_{3}, \\ J f_{j}(\omega) & \text { if } \omega \in \varsigma(I) \cap E .\end{cases}
$$

Then $F_{j}(\omega) \in L^{\infty}(\tilde{\gamma})$ and for $z \in \tilde{D}$,

$$
f_{j}(z)=\frac{1}{2 \pi i} \int_{\tilde{\gamma}} \frac{F_{j}(\omega)}{\omega-z} d \omega,
$$

$F_{j}(\omega)$ induces a function $F_{j}(\omega(\xi))$ on $\gamma$. For $z \in D$ we set

$$
f_{j}^{*}(z)=\frac{1}{2 \pi i} \int_{\gamma} \frac{F_{j}(\omega(\xi))(d \omega / d \xi)(\xi)}{\xi-z} d \xi
$$

Then by Lemma 2 , if $z=x+i y \in D_{1 / 2}$,

$$
\left|\frac{1}{\zeta^{\prime}(x)} f_{j}^{*}(z)-f_{j}(T z)\right| \leq C\left\|F_{j} \frac{d \omega}{d \xi}\right\|_{\infty}\left(|I|^{\alpha}+\frac{\delta}{|I|}+\frac{|I|}{\delta} \beta\right) .
$$

Since $\left\|F_{j} d \omega / d \xi\right\|_{\infty} \leq 2$, we can make the right-hand side of this inequality small by first assuming that $\delta$ is some small fraction of $|I|$, and then assuming that $|I|$ and hence $\beta \leq c|I|^{\alpha}$ are small. Assuming that the right-hand side of (8) is less than $\mu / 2$, it then follows that $\mu / 2 \leq \max \left\{\left|f_{j}^{*}(z)\right|: 1 \leq j \leq n\right\} \leq 1+\mu / 2$ for $z \in D_{1 / 2}$. Since $D_{1 / 2}$ is a Denjoy domain (actually it is conformally equivalent to a Denjoy domain), 
then by the theorem of Garnett and Jones, there exists $h_{1}^{*}, \ldots, h_{n}^{*} \in H^{\infty}\left(D_{1 / 2}\right)$ such that $f_{1}^{*} h_{1}^{*}+\cdots+f_{n}^{*} h_{n}^{*} \equiv 1$ on $D_{1 / 2}$.

Completely analogous to the relationship between $D$ and $\tilde{D}$, we form the domain $\tilde{D}_{1 / 2}$ corresponding to $D_{1 / 2}$. We then let $\gamma_{1 / 2}=\partial D_{1 / 2}, \tilde{\gamma}_{1 / 2}=\partial \tilde{D}_{1 / 2}$ and we define $\omega_{1 / 2}: \gamma_{1 / 2} \rightarrow \tilde{\gamma}_{1 / 2}$ in the same way we defined $\omega$. Also, we will let $I_{1 / 2}$ denote the interval centered at $s_{0}$ and having length $|I| / 2=(b-a) / 2$, and we set

$$
D_{1 / 4}=\left\{x+i y:\left|x-s_{0}\right|<\frac{b-a}{8},|y|<\frac{\delta}{4}\right\}-\varsigma^{-1}(E) .
$$

Define functions $H_{j}^{*}(\xi) \in L^{\infty}\left(\gamma_{1 / 2}\right), 1 \leq j \leq n$, by

$$
H_{j}^{*}(\xi)= \begin{cases}h_{j}^{*}(\xi) & \text { if } \xi \in \gamma_{1 / 2}-\varsigma^{-1}(E), \\ J h_{j}^{*}(\xi) & \text { if } \xi \in I_{1 / 2} \cap \varsigma^{-1}(E) .\end{cases}
$$

Then for $z \in D_{1 / 2}, h_{j}^{*}(z)=(1 / 2 \pi i) \int_{\gamma_{1 / 2}} H_{j}^{*}(\xi) /(\xi-z) d \xi$. For $z \in \tilde{D}_{1 / 2}$ define $h_{j}(z)=(1 / 2 \pi i) \int_{\gamma_{1 / 2}} H_{j}^{*}(\xi) /\left(\omega_{1 / 2}(\xi)-z\right) d \xi$. Then by Lemma 2 , if $z=x+i y \in$ $D_{1 / 4}$,

$$
\left|\frac{1}{\zeta^{\prime}(x)} h_{j}^{*}(z)-h_{j}(T z)\right| \leq c|| h_{j} \|_{\infty}\left(\left|I_{1 / 2}\right|^{\alpha}+\frac{\delta / 2}{\left|I_{1 / 2}\right|}+\frac{2\left|I_{1 / 2}\right|_{\beta}}{\delta}\right) .
$$

Then, by choosing $\delta$ and $|I|$ in the manner described previously, we can assume that the right-hand sides of (8) and (9) are sufficiently small so that for $z=x+i y \in D_{1 / 4}$,

$$
\begin{aligned}
& \left.|| \sum_{j=1}^{n} f_{j}(T z) h_{j}(T z)\right]-\left(\frac{1}{\zeta^{\prime}(x)}\right)^{2} \mid \\
& \quad=\left|\sum_{j=1}^{n} f_{j}(T z) h_{j}(T z)-\frac{f_{j}^{*}(z) h_{j}^{*}(z)}{\left(\zeta^{\prime}(x)\right)^{2}}\right| \\
& \quad \leq \sum_{j=1}^{n}\left(\left|f_{j}(T z) h_{j}(T z)-f_{j}(T z) \frac{h_{j}^{*}(z)}{\zeta^{\prime}(x)}\right|+\left|f_{j}(T z) \frac{h_{j}^{*}(z)}{\zeta^{\prime}(x)}-\frac{f_{j}^{*}(z)}{\zeta^{\prime}(x)} \frac{h_{j}^{*}(z)}{\zeta^{\prime}(x)}\right|\right)<\frac{1}{2} .
\end{aligned}
$$

Then we set $U=T\left(D_{1 / 4}\right)$. $U$ is a neighborhood of $\varsigma\left(s_{0}\right)$, and the functions $g_{j}(z)=$ $h_{j}(z) /\left(\sum_{j=1}^{n} f_{j}(z) h_{j}(z)\right)$ are in $H^{\infty}(U)$ and satisfy $f_{1} g_{1}+\cdots+f_{n} g_{n} \equiv 1$ on $U$.

\section{REFERENCES}

1. T. W. Gamelin, Localization of the corona problem, Pacific J. Math. 34 (1970), 73-81.

2. J. B. Garnett, Bounded analytic functions, Academic Press, New York, 1981.

3. J. B. Garnett and P. W. Jones, The corona theorem for Denjoy domains, Acta Math. 155 (1985), 27-40.

Department of Mathematics, University of California, los Angeles, CaliFORNIA 90024 63130

Current address: Department of Mathematics, Washington University, St. Louis, Missouri 